PROCEEDINGS OF THE

AMERICAN MATHEMATICAL SOCIETY

Volume 135, Number 1, January 2007, Pages 191-200

S 0002-9939(06)08468-

Article electronically published on June 22, 2006

\title{
SINGLE ELEMENTS AND MODULE ISOMORPHISMS OF SOME OPERATOR ALGEBRA MODULES
}

\author{
DONG ZHE
}

(Communicated by David R. Larson)

\begin{abstract}
In this paper, we first introduce the concept of single elements in a module. A systematic study of single elements in the $\operatorname{Alg} \mathcal{L}$-module $\mathcal{U}$ is initiated, where $\mathcal{L}$ is a completely distributive subspace lattice on a Hilbert space $\mathcal{H}$. Furthermore, as an application of single elements, we study module isomorphisms between norm closed $\operatorname{Alg} \mathcal{N}$-modules, where $\mathcal{N}$ is a nest, and obtain the following result: Suppose that $\mathcal{U}, \mathcal{V}$ are norm closed $\operatorname{Alg} \mathcal{N}$-modules and that $\Phi: \mathcal{U} \rightarrow \mathcal{V}$ is a module isomorphism. Then $\mathcal{U}=\mathcal{V}$ and there exists a non-zero complex number $\lambda$ such that $\Phi(T)=\lambda T, \forall T \in \mathcal{U}$.
\end{abstract}

\section{INTRODUCTION AND PRELIMINARIES}

An element $S$ of an algebra $\mathcal{A}$ is called single if the condition $A S B=0$ for $A, B$ in $\mathcal{A}$ implies $A S=0$ or $S B=0$. It is easy to show that a rank one operator is a single element of any operator algebra containing it. It is primarily for this reason that the notion of 'single element' plays a role in the representation theory of $C^{*}$-algebras or, more generally, of semi-simple Banach algebras ([2, 3]). In yet another aspect, namely in the study of algebraic isomorphisms between reflexive operator algebras on a normed space, single elements have also proved a useful tool. This is mainly because single elements are carried to single elements under algebraic isomorphisms. So if, in a particular operator algebra, it is shown that each single element is of rank one (the converse is always true), then the study of algebraic isomorphism is considerably simplified. Reflexive algebras that have been looked at from this point of view include nest algebras on a Hilbert space [9] and algebras of operators leaving invariant the elements of a complete atomic Boolean lattice of subspaces on normed space [5]. On each of the two above-mentioned operator algebras, it is proved in [5], 9] that single elements are of rank one. Using this, one shows that each algebraic isomorphism between a pair of such algebras is automatically continuous and spatial in the sense that it is of the form $\Phi(A)=T^{-1} A T$ for suitable $T([5],[9])$.

A systematic study of single elements of a reflexive operator algebra $\operatorname{Alg} \mathcal{L}$, where $\mathcal{L}$ is completely distributive, was initiated by Lambrou in $[\underline{6}$. Amongst many other interesting results, he shows that single elements of any rank (including infinity)

Received by the editors November 3, 2004 and, in revised form, July 30, 2005.

2000 Mathematics Subject Classification. Primary 47L75.

Key words and phrases. Single element, module isomorphism, nest algebra module.

This project was partially supported by the National Natural Science Foundation of China (No. 10401030), the Zhejiang Natural Science Foundation (No. M103044) and GFJG.

(C)2006 American Mathematical Society 191

Reverts to public domain 28 years from publication 
are possible. In [8], Longstaff and Penaia obtained a lattice-theoretic condition for the existence of a single element of rank $n$ (or infinity).

Definition 1.1. Let $\mathcal{R}$ be a ring and $\mathcal{M}$ a $\mathcal{R}$-bimodule. An element $s$ in $\mathcal{M}$ is called single if, whenever $a s b=0$ with $a, b \in \mathcal{R}$, then $a s=0$ or $s b=0$.

Though this is a simple generalization of the notion of single elements in algebras, we hope that as single elements in algebras, single elements in modules will play a role in the representation theory of modules, and in the study of module isomorphisms between modules, especially in operator module theory. In Section 2 of this paper, we initiate the study of single elements in $\operatorname{Alg} \mathcal{L}$-modules, where $\mathcal{L}$ is a completely distributive subspace lattice on a Hilbert space $\mathcal{H}$, and we obtain some elementary properties of single elements in $A \lg \mathcal{L}$-modules. As a corollary, we show that every single element of nest algebra modules is of rank one. In Section 3 , using single elements of nest algebra modules, we completely solve the problem of module isomorphisms between norm closed nest algebra modules. It turns out that such isomorphisms are necessarily trivial.

Let us introduce some notation and terminology. $\mathcal{H}$ represents a complex separable infinite-dimensional Hilbert space, and $\mathcal{B}(\mathcal{H})$ the algebra of all bounded operators on $\mathcal{H}$. The term 'subspace' of $\mathcal{H}$ shall mean a closed linear manifold of $\mathcal{H}$. A collection $\mathcal{L}$ of subspaces of $\mathcal{H}$ is called a subspace lattice on $\mathcal{H}$ if it contains $(0)$ and $\mathcal{H}$, and is closed under the formation of arbitrary closed linear spans (denoted ' $\vee$ ') and intersection (denoted ' $\cap$ '). If in a subspace lattice $\mathcal{L}$ the infinite distributive identity

$$
\bigcap_{a \in A} \bigvee_{b \in B} L_{a, b}=\bigvee_{f \in B^{A}} \bigcap_{a \in A} L_{a, f(a)}
$$

and its dual holds, $\mathcal{L}$ is called completely distributive. The formal definition of complete distributivity just given is in practice and difficult to use. Alternative characterizations of complete distributivity have been proven to be more useful.

Lemma 1.1 ([7]). For a subspace lattice $\mathcal{L}$, the following are equivalent:

(1) $\mathcal{L}$ is completely distributive;

(2) $L=\bigcap\left\{K_{-}: K \in \mathcal{L}, K \nsubseteq L\right\}$, for every $L \in \mathcal{L}$;

(3) $L=\bigvee\left\{K \in \mathcal{L}: K_{-} \nsupseteq L\right\}$, for every $L \in \mathcal{L}$; here $K_{-}=\bigvee\{G \in \mathcal{L}: G \nsupseteq K\}$.

It follows from Lemma 1.1(3) that the linear manifold $\mathcal{H}_{0}=\operatorname{span}\{K \in \mathcal{L}$ : $\left.K \neq(0), K_{-} \neq \mathcal{H}\right\}$ is norm dense in $\mathcal{H}$. Similarly, Lemma 1.1(2) shows that $\mathcal{H}_{1}=\operatorname{span}\left\{K_{-}^{\perp}: K \neq(0), K_{-} \neq \mathcal{H}\right\}$ is also norm dense in $\mathcal{H}$.

If $\mathcal{L}$ is a subspace lattice, $\operatorname{Alg} \mathcal{L}$ denotes the set of operators on $\mathcal{H}$ leaving every member of $\mathcal{L}$ invariant. Obviously, $\operatorname{Alg} \mathcal{L}$ is a unital Banach algebra. Operator algebras of the type $\operatorname{Alg} \mathcal{L}$ are called reflexive operator algebras. A nest $\mathcal{L}$ is a totally ordered subspace lattice, and $\operatorname{Alg} \mathcal{L}$ is called the nest algebra associated with the nest $\mathcal{L}$.

If $x$ and $y$ are non-zero vectors in $\mathcal{H}$, we define the rank one operator $x \otimes y$ by

$$
(x \otimes y)(z)=(z, y) x, \quad \forall z \in \mathcal{H} .
$$

Lemma $1.2([7])$. If $\mathcal{L}$ is a subspace lattice, then the rank one operator $x \otimes y$ belongs to $A l g \mathcal{L}$ if and only if there is an element $L \in \mathcal{L}$ such that $x \in L$ and $y \in L_{-}^{\perp}$, where $L_{-}=\bigvee\{K \in \mathcal{L}: K \nsupseteq L\}$. 
Definition 1.2. Let $\mathcal{L}$ be a subspace lattice and let $\mathcal{U}, \mathcal{V}$ be $\operatorname{Alg} \mathcal{L}$-bimodules. A module homomorphism $\Phi$ from $\mathcal{U}$ to $\mathcal{V}$ is a linear map $\Phi: \mathcal{U} \rightarrow \mathcal{V}$ such that

$$
\Phi(A T B)=A \Phi(T) B, \quad \forall A, B \in \operatorname{Alg} \mathcal{L}, T \in \mathcal{U} .
$$

Furthermore, if $\Phi$ is a bijection, we shall say that $\Phi$ is a module isomorphism.

In Section 3 of this paper, we will investigate the problem of the module isomorphism between norm closed nest algebra modules. The terminology and notation concerning nest algebra modules may be found in [4].

\section{Single elements in Alg $\mathcal{L}$-modules}

Lemma 2.1. Let $\mathcal{L}$ be a completely distributive subspace lattice on $\mathcal{H}$, and let $A \in \mathcal{B}(H)$.

(1) If $R A=0$ for all rank one operators $R \in A l g \mathcal{L}$, then $A=0$.

(2) If $A R=0$ for all rank one operators $R \in A l g \mathcal{L}$, then $A=0$.

Proof. (1) If $R \in \operatorname{Alg} \mathcal{L}$ is of rank one, then $R=x \otimes y$, where $y \in K_{-}^{\perp}$ for some $K \in \mathcal{L}$. The condition $0=(x \otimes y) A=x \otimes A^{*} y$ for all rank ones of $\operatorname{Alg} \mathcal{L}$ implies ker $A^{*} \supseteq K_{-}^{\perp}$, so taking the linear span over all such $K$, we have $\operatorname{ker} A^{*} \supseteq \mathcal{H}_{1}$, where $\mathcal{H}_{1}$ is as defined just below the statement of Lemma 1.1. The continuity of $A^{*}$ and the norm density of $\mathcal{H}_{1}$ implies that $A^{*}$, and hence $A$, is zero.

(2) The condition $0=A(x \otimes y)=A x \otimes y$ implies ker $A \supseteq K$ for each $K$ with $K_{-} \neq \mathcal{H}$. So $\operatorname{ker} A \supseteq \mathcal{H}_{0}$, which is norm dense in $\mathcal{H}$, and so $A$ is zero.

Lemma 2.2. Let $\mathcal{L}$ be a complete distributive subspace lattice and $\mathcal{U}$ an $A l g \mathcal{L}$ module. Then an element $S$ of $\mathcal{U}$ is single if and only if for each rank one operator $R_{1}, R_{2}$ of AlgL, the condition $R_{1} S R_{2}=0$ implies that $R_{1} S$ or $S R_{2}$ is zero.

Proof. If $S$ is single, then the above condition is only a special case of the definition. Suppose that $A S B=0$ for $A, B \in \operatorname{Alg} \mathcal{L}$. If $S B \neq 0$, then by Lemma 2.1 there exists a rank one operator $R_{2}$ of $\operatorname{Alg} \mathcal{L}$ such that $S B R_{2} \neq 0$. For any rank one $R_{1}$ of $\operatorname{Alg} \mathcal{L}$, we have $R_{1} A S B R_{2}=0$, and clearly $R_{1} A$ and $B R_{2}$ are of rank one or zero. In either case the condition in the lemma implies $R_{1} A S$ or $S B R_{2}$ is zero. But as $S B R_{2} \neq 0$, we have for all rank one operators $R_{1}$ of $\operatorname{Alg} \mathcal{L}$ that $R_{1} A S$ is zero. Applying Lemma 2.1 once again, it follows that $A S=0$, and this shows that $S$ is a single element of $\mathcal{U}$.

Lemma 2.3. Let $\mathcal{L}$ be a completely distributive subspace lattice, $\mathcal{U}$ an Alg $\mathcal{L}$-module, and $S$ a single element of $\mathcal{U}$. Then there exists an $M$ in $\mathcal{L}$ with $M_{-} \neq \mathcal{H}$ such that $\left.S\right|_{M}$ is non-zero. Moreover, for any $L \in \mathcal{L}$ with $L_{-} \neq \mathcal{H}$ and $\left.S\right|_{L}$ non-zero, the operator $\left.S\right|_{L}$ is of rank one.

Proof. By Lemma 2.1 there is a rank one operator $R \in \operatorname{Alg} \mathcal{L}$ such that $S R \neq 0$. By Lemma 1.2, $R$ is of the form $x \otimes y$ where $x \in M, y \in M_{-}^{\perp}$ for some $M \in \mathcal{L}$ and $M_{-} \neq \mathcal{H}$. But then $0 \neq S(x \otimes y)=S x \otimes y$ shows that $S x \neq 0$, and the first part of the lemma is proved.

By Lemma 2.1 there is a rank one $T \in \operatorname{Alg} \mathcal{L}$ such that $T S \neq 0$. Now let $L \in \mathcal{L}$ satisfy the condition in the statement of the lemma. We are to prove that if $x, y \in L$, then $S x$ and $S y$ are linearly dependent, so there is no loss in assuming that $S x, S y$ are non-zero. 
The operator $T S$ is of rank one, so there exist scalars $\lambda, \mu$ not both zero, such that $T S(\lambda x+\mu y)=\lambda(T S x)+\mu(T S y)=0$. For any non-zero $z \in L_{-}^{\perp}$, we have $(\lambda x+\mu y) \otimes z \in \operatorname{Alg} \mathcal{L}$ and

$$
T S[(\lambda x+\mu y) \otimes z]=T S(\lambda x+\mu y) \otimes z=0 .
$$

However, $S$ is single and $T S \neq 0$, so

$$
S(\lambda x+\mu y) \otimes z=S[(\lambda x+\mu y) \otimes z]=0,
$$

which in turn implies $\lambda(S x)+\mu(S y)=0$, and so $S x, S y$ are linearly dependent. Thus the operator $\left.S\right|_{L}$ is of rank one.

Suppose that $\mathcal{L}$ is a completely distributive subspace lattice; for any $L \in \mathcal{L}$ we set $\tau(L)=[\mathcal{U} L]$. Since $\mathcal{L}$ is completely distributive, by virtue of Lemma 1.1 and Lemma 1.2 we can easily show that $\mathcal{L}=\operatorname{LatAlg} \mathcal{L}$. Thus $\tau(L)=[\mathcal{U} L] \in \operatorname{LatAlg} \mathcal{L}=$ $\mathcal{L}$, and $\tau$ is an order homomorphism from $\mathcal{L}$ into itself. Define

$$
\mathcal{U}_{\tau}=\{T \in \mathcal{B}(H): T L \subseteq \tau(L), \forall L \in \mathcal{L}\} .
$$

Clearly $\mathcal{U}_{\tau} \supseteq \mathcal{U}$ and $\mathcal{U}_{\tau}$ is a weakly closed $\operatorname{Alg} \mathcal{L}$-module.

Proposition 2.1. Let $\mathcal{L}$ be a completely distributive subspace lattice, $\mathcal{U}$ an AlgLmodule and $S$ a non-zero single element of $\mathcal{U}$. If $\left.S\right|_{M}$ non-zero for an $M \in \mathcal{L}$ with $M_{-} \neq \mathcal{H}$, then $S(\mathcal{H}) \subseteq \tau(M)=[\mathcal{U} M]$.

Proof. Let $L \in \mathcal{L}$ with $L \nsubseteq \tau(M)$. We shall first show that $S(\mathcal{H}) \subseteq L_{-}$. If $L_{-}=\mathcal{H}$ we have nothing to prove, so assume $L_{-} \neq \mathcal{H}$. The condition $L \nsubseteq \tau(M)$ implies $\tau(M) \subseteq L_{-}$, so if $m \in M$, it follows from $S \in \mathcal{U} \subseteq \mathcal{U}_{\tau}$ that

$$
S m \in S(M) \subseteq \tau(M) \subseteq L_{-} .
$$

Now let $y \in L_{-}^{\perp}$ be arbitrary. Choose non-zero $x \in L$ and $m \in M$ with $S m \neq 0$ and non-zero $l \in M_{-}^{\perp}$. Then by Lemma 1.2 the rank one $x \otimes y$ and $m \otimes l$ belong to $\operatorname{Alg} \mathcal{L}$, and $S(m \otimes l) \neq 0$. However, $S m \in L_{-}$and $y \in L_{-}^{\perp}$, so

$$
(x \otimes y) S(m \otimes l)=(x \otimes y)(S m \otimes l)=(S m, y) x \otimes l=0 .
$$

The assumption that $S$ is single implies $(x \otimes y) S=0$. Thus for any $h \in \mathcal{H}$, we have

$$
(x \otimes y) S h=(S h, y) x=0,
$$

and so $(S h, y)=0$ for any $y \in L_{-}^{\perp}$. This shows that $S h \in L_{-}$and $S(\mathcal{H}) \subseteq L_{-}$, as required.

Since $\mathcal{L}$ is completely distributive, it follows from Lemma 1.1 that $\tau(M)=$ $\bigcap\left\{L_{-}: L \in \mathcal{L}, L \nsubseteq \tau(M)\right\}$. Hence $S(\mathcal{H}) \subseteq \bigcap\left\{L_{-}: L \in \mathcal{L}, L \nsubseteq \tau(M)\right\}=\tau(M)$.

For an $\operatorname{Alg} \mathcal{L}$-module $\mathcal{U}$, we define

$$
\overline{\mathcal{U}}_{\tau}=\{T \in \mathcal{B}(H): T \tau(L) \subseteq L, \forall L \in \mathcal{L}\} .
$$

Theorem 2.1. Let $\mathcal{L}$ be a completely distributive subspace lattice and $\mathcal{U}$ an AlgLmodule. If $S$ is a single element of $\mathcal{U}$ with $S \overline{\mathcal{U}}_{\tau} S \neq 0$, then $S$ is of rank one.

Proof. Let $A \in \overline{\mathcal{U}}_{\tau}$ be such that $S A S \neq 0$, and let $l_{2}$ be such that $S A S l_{2} \neq 0$. Put $l_{1}=A S l_{2}$ and $l=S l_{1}$. We will show that $S(\mathcal{H}) \subseteq \mathbf{C l}$. First we show that if $K \in \mathcal{L}$ with $K_{-} \neq \mathcal{H}$, then $S(K) \subseteq \mathbf{C l}$. Indeed if $S(K)=\{0\}$ we have nothing to prove. If instead $S(K) \neq\{0\}$, Proposition 2.1 implies that $S(\mathcal{H}) \subseteq \tau(K)$. But then $l_{1}=A S l_{2} \in A(\tau(K)) \subseteq K$, since $A \in \overline{\mathcal{U}}_{\tau}$. Note that $\left.S\right|_{K}$ is non-zero, since $l_{1} \in K$ and $S l_{1}=S A S l_{2} \neq 0$; thus Lemma 2.3 implies $\left.S\right|_{K}$ is of rank one. 
Hence $S(K) \subseteq \mathbf{C} S l_{1}=\mathbf{C l}$, as claimed. Now denoting by $\mathcal{H}_{0}$ the linear span of $\left\{K \in \mathcal{L}: K \neq(0), K_{-} \neq \mathcal{H}\right\}$, the above also shows that $S\left(\mathcal{H}_{0}\right) \subseteq \mathbf{C l}$. But it follows from Lemma 1.1 that $\mathcal{H}_{0}$ is dense in $\mathcal{H}$, so

$$
S(\mathcal{H})=S\left(\overline{\mathcal{H}_{0}}\right) \subseteq \overline{S\left(\mathcal{H}_{0}\right)} \subseteq \overline{\mathbf{C l}}=\mathbf{C l}
$$

This completes the proof.

Obviously, nests are completely distributive, so the results in the section also hold for nests.

Corollary 2.1. If $\mathcal{L}$ is a nest and $\mathcal{U}$ is an Alg $\mathcal{L}$-module, then every non-zero single element of $\mathcal{U}$ is of rank one, and conversely.

Proof. Let $S$ be a single element of $\operatorname{Alg} \mathcal{L}$. By Lemma 2.3 that there exists an $L$ in $\mathcal{L}$ such that $S(L)$ is one dimensional. Let $M \in \mathcal{L}, M_{-} \neq \mathcal{H}$ with $M \supseteq L$. Since $S(M)$ is non-zero, it follows from Lemma 2.3 again that $S(M)$ is also one dimensional. Clearly the one-dimensional subspaces $S(M), S(L)$ coincide, as $L \subseteq M$. Now, by the total order of $\mathcal{L}$, the set $\mathcal{H}_{0}=\bigcup\left\{M \in \mathcal{L}: M_{-} \neq \mathcal{H}, M \supseteq L\right\}$ is a dense linear manifold, and by the above $S\left(\mathcal{H}_{0}\right)$ is one dimensional, thus $S\left(\mathcal{H}_{0}\right)=\mathbf{C l}$ for some non-zero vector $l \in \mathcal{H}$. Hence, $S(\mathcal{H})=\mathbf{C} l$ and $S$ is of rank one.

\section{Module ISOMORPHISMS}

In this section, we are concerned with the problem of module isomorphisms between nest algebra modules. Since one feels that non-norm-closed nest algebra modules are rather pathological and that the proper objects for study should at least be complete, we only concern ourselves with norm closed nest algebra modules. In this section, we adopt the terminology and notation in [4. $\mathcal{N}$ always represents a nest on $\mathcal{H}$, and $\mathcal{U}$ is a norm closed $\operatorname{Alg} \mathcal{N}$-module. In the following, we set $\tau(N)=\widetilde{N}=[\mathcal{U} N]$ for any $N \in \mathcal{N}$ and $\mathcal{U}_{\tau}=\{T \in \mathcal{B}(H): T N \subseteq \widetilde{N}, \forall N \in \mathcal{N}\}$.

Lemma 3.1. Suppose that $\mathcal{U}, \mathcal{V}$ are norm closed AlgN-modules, and that $\Phi: \mathcal{U} \rightarrow \mathcal{V}$ is a module isomorphism. Then $\Phi$ preserves rank one operators; furthermore, $\Phi$ preserves rank.

Proof. Let $T \in \mathcal{U}$ have rank one. It follows from Corollary 2.1 that we only need to show that $\Phi(T)$ is a single element of $\mathcal{V}$. Let $A, B \in \operatorname{Alg} \mathcal{N}$ with $A \Phi(T) B=0$. Since $0=A \Phi(T) B=\Phi(A T B)$ and $\Phi$ is a module isomorphism, it follows that $A T B=0$. Since $T$ is of rank one, at least one of $A T, T B$ is zero. Thus, it follows from $A \Phi(T)=\Phi(A T)$ and $\Phi(T) B=\Phi(T B)$ that at least one of $A \Phi(T), \Phi(T) B$ is zero. Therefore $\Phi(T)$ is single in $\mathcal{V}$ and from Corollary 2.1, $\Phi(T) \in \mathcal{V}$ is a rank one operator.

Set $\tau(N)=[\mathcal{U} N]=\widetilde{N}$ for every $N \in \mathcal{N}$ and $\mathcal{U} \subseteq \mathcal{U}_{\tau}=\{T \in \mathcal{B}(H): T N \subseteq$ $\tilde{N}, \forall N \in \mathcal{N}\}$. It follows from [4], Lemma 1.3, that $\mathcal{U}_{\tau}$ and $\mathcal{U}$ contain the same set of operators of rank one. Hence from [4, Lemma 1.2, each rank $n$ operator in $\mathcal{U}$ may be written as the sum of $n$ elements of $\mathcal{U}$ each having rank one, so $\Phi$ preserves rank.

Let $\mathcal{U}$ be a norm closed $\operatorname{Alg} \mathcal{N}$-module, and let $\mathcal{O}(\mathcal{U})$ be the set of rank one operators in $\mathcal{U}$. It is easy to see from Proposition 3.1 that $\Phi(\mathcal{O}(\mathcal{U}))=\mathcal{O}(\mathcal{V})$. 
Lemma 3.2. Suppose that $\mathcal{U}_{i}(i=1,2,3)$ are norm closed $A \lg \mathcal{N}$-modules, and that $\Phi: \mathcal{U}_{1} \rightarrow \mathcal{U}_{2}, \Psi: \mathcal{U}_{1} \rightarrow \mathcal{U}_{3}$ are module isomorphisms. If for each rank one element $S$ of $\mathcal{U}_{1}, \Phi(S)=\Psi(S)$, then $\mathcal{U}_{2}=\mathcal{U}_{3}$ and $\Phi=\Psi$.

Proof. Suppose that there exists $T \in \mathcal{U}_{1}$, such that $\Phi(T) \neq \Psi(T)$. Set $T^{\prime}=$ $\Phi(T)-\Psi(T) \neq 0$. From Lemma 2.1, there exists a rank one operator $S$ of $\operatorname{Alg} \mathcal{N}$ such that $S T^{\prime} \neq 0$. Since $\operatorname{dim} \operatorname{ran}(S T) \leq 1$, we have

$$
S T^{\prime}=S \Phi(T)-S \Psi(T)=\Phi(S T)-\Psi(S T)=0,
$$

which is a contradiction. Hence, for each $T \in \mathcal{U}_{1}, \Phi(T)=\Psi(T)$ and $\mathcal{U}_{2}=\mathcal{U}_{3}$.

In the following, let $\mathcal{A}$ be maximal abelian *-subalgebra of $\operatorname{Alg} \mathcal{N}$. It follows from 9], Lemma 2.8, that $\mathcal{A} \supseteq\{P(N): N \in \mathcal{N}\}$ and $\mathcal{A}$ is a maximal abelian ${ }^{*}$-subalgebra of $\mathcal{B}(H)$. For a norm closed $\operatorname{Alg} \mathcal{N}$-module $\mathcal{U}$, we set $\mathcal{N}_{0}=\left\{N \in \mathcal{N}: N \neq(0), N_{\sim} \neq\right.$ $\mathcal{H}\}$. Recall that $N_{\sim}=\bigvee\left\{N^{\prime} \in \mathcal{N}: \widetilde{N^{\prime}}<N\right\}$ and $\widetilde{N^{\prime}}=\left[\mathcal{U} N^{\prime}\right]=\tau\left(N^{\prime}\right)$.

Lemma 3.3. Suppose that $\mathcal{U}, \mathcal{V}$ are norm closed AlgN-modules. If $\Phi: \mathcal{U} \rightarrow \mathcal{V}$ is a module isomorphism, then for $N \in \mathcal{N}_{0}$ and non-zero vectors $x_{1}, x_{2} \in N, y \in N_{\sim}^{\perp}$, there exist non-zero vectors $u_{i} \in N, v \in N_{\sim}^{\perp}$ such that

$$
\Phi\left(x_{i} \otimes y\right)=u_{i} \otimes v, \quad i=1,2 .
$$

Proof. From 4, Lemma 1.1, $x_{i} \otimes y \in \mathcal{U}_{\tau}$. It follows from 4, Lemma 1.3, that $\mathcal{U}_{\tau}$ and $\mathcal{U}$ contain the same set of operators of rank one, so $x_{i} \otimes y \in \mathcal{U}$. Since $\Phi$ is a module isomorphism, it follows from Lemma 3.1 that there exist non-zero vectors $u_{i}, v_{i}(i=1,2)$ such that

$$
\Phi\left(x_{i} \otimes y\right)=u_{i} \otimes v_{i}, \quad i=1,2 .
$$

Since

$$
\begin{aligned}
u_{i} \otimes v_{i} & =\Phi\left(x_{i} \otimes y\right)=\Phi\left(P(N)\left(x_{i} \otimes y\right) P\left(N_{\sim}\right)^{\perp}\right) \\
& =P(N) \Phi\left(x_{i} \otimes y\right) P\left(N_{\sim}\right)^{\perp}=P(N) u_{i} \otimes P\left(N_{\sim}\right)^{\perp} v_{i}
\end{aligned}
$$

we may assume $u_{i} \in N, v_{i} \in N_{\sim}^{\perp}$.

It follows from [9], Lemma 2.11, that there exist $A_{i} \in \mathcal{A} \subseteq \operatorname{Alg} \mathcal{N}, x \in \mathcal{H}$ such that $A_{i} x=x_{i}$. Since

$$
x_{i}=P(N) x_{i}=P(N) A_{i} x=A_{i} P(N) x,
$$

we may assume $x \in N$ (if not, replace $x$ by $P(N) x$; since $P(N)$ and $A_{i}$ are members of $\mathcal{A}$, and thus commute, the required conditions are still satisfied) and $\Phi(x \otimes y)=$ $u_{3} \otimes v_{3}$. Thus, we have that

$$
\Phi\left(x_{i} \otimes y\right)=\Phi\left(A_{i} x \otimes y\right)=A_{i} \Phi(x \otimes y)=A_{i} u_{3} \otimes v_{3} .
$$

From (1) and (2), $v_{1}, v_{2}$ are linearly dependent. This completes the proof.

Lemma 3.4. Suppose that $\mathcal{U}, \mathcal{V}$ are norm closed AlgN-modules. If $\Phi: \mathcal{U} \rightarrow \mathcal{V}$ is a module isomorphism, then for each $N \in \mathcal{N}_{0}$, there exist injective linear maps $U_{N}: N \rightarrow N$ and $V_{N}: N_{\sim}^{\perp} \rightarrow N_{\sim}^{\perp}$ such that

$$
\Phi(x \otimes y)=U_{N} x \otimes V_{N} y, \quad \forall x \in N, y \in N_{\sim}^{\perp} .
$$


Proof. For each $0 \neq y \in N_{\sim}^{\perp}$, it follows from Lemma 3.3 that there exists an injective linear map $U_{y}: N \rightarrow N$ and a non-zero vector $v_{y} \in N_{\sim}^{\perp}$ such that

$$
\Phi(x \otimes y)=U_{y} x \otimes v_{y}, \quad \forall x \in N .
$$

Thus, for $x \in N, y_{i} \in N_{\sim}^{\perp}(i=1,2)$, we have that

$$
\Phi\left(x \otimes y_{i}\right)=U_{y_{i}} x \otimes v_{y_{i}} .
$$

From [9, Lemma 2.11, there exists $A_{i} \in \mathcal{A} \subseteq \operatorname{Alg} \mathcal{N}$ and $y_{0} \in \mathcal{H}$ such that $A_{i} y_{0}=y_{i}$. Since

$$
y_{i}=P\left(N_{\sim}\right)^{\perp} y_{i}=P\left(N_{\sim}\right)^{\perp} A_{i} y_{0}=A_{i} P\left(N_{\sim}\right)^{\perp} y_{0},
$$

we may assume that $y_{0} \in N_{\sim}^{\perp}$ and $\Phi\left(x \otimes y_{0}\right)=a \otimes b_{0}$, where $a \in N$ and $b_{0} \in N_{\sim}^{\perp}$. Then we have that

$$
\Phi\left(x \otimes y_{i}\right)=\Phi\left(x \otimes A_{i} y_{0}\right)=\Phi\left(x \otimes y_{0}\right) A_{i}^{*}=a \otimes A_{i} b_{0}, \quad i=1,2 .
$$

By virtue of (4) and (5), we can show that there exists a non-zero complex number $\lambda$ (independent of $x$ ) such that

$$
U_{y_{1}} x=\lambda U_{y_{2}} x, \quad \forall x \in N .
$$

Indeed, it follows from (4) and (5) that

$$
U_{y_{1}} x \otimes v_{y_{1}}=a \otimes A_{1} b_{0}
$$

and

$$
U_{y_{2}} x \otimes v_{y_{2}}=a \otimes A_{2} b_{0} .
$$

Thus there exist non-zero complex numbers $\lambda_{i}, \kappa_{i}(i=1,2)$ such that

$$
U_{y_{1}} x=\lambda_{1} a, v_{y_{1}}=\kappa_{1} A_{1} b_{0} \text { and } \lambda_{1} \bar{\kappa}_{1}=1,
$$

and

$$
U_{y_{2}} x=\lambda_{2} a, v_{y_{2}}=\kappa_{2} A_{2} b_{0} \text { and } \lambda_{2} \bar{\kappa}_{2}=1
$$

So

$$
U_{y_{1}} x=\lambda_{1} a=\frac{\lambda_{1}}{\lambda_{2}} \lambda_{2} a=\frac{\lambda_{1}}{\lambda_{2}} U_{y_{2}} x=\left(\frac{\overline{\kappa_{2}}}{\kappa_{1}}\right) U_{y_{2}} x .
$$

Since $v_{y_{i}}$ and $A_{i} b_{0}$ are independent of $x$, so $\kappa_{i}(i=1,2)$ are independent of $x$. Let $\lambda=\left(\frac{\frac{y_{2}}{\kappa_{2}}}{\kappa_{1}}\right.$. So there exists a non-zero complex number $\lambda$ (independent of $x$ ) such that $U_{y_{1}}=\lambda U_{y_{2}}$. Hence, if a suitable normalization is chosen, we may assume that $U_{y}$ is in fact independent of $y$, and write $U_{N}$ for $U_{y}$. Thus, (3) becomes

$$
\Phi(x \otimes y)=U_{N} x \otimes v_{y}, \quad \forall x \in N, y \in N_{\sim}^{\perp} .
$$

For each $0 \neq y \in N_{\sim}^{\perp}$, we have that $0 \neq v_{y} \in N_{\sim}^{\perp}$ and $v_{y}$ depends linearly on $y$. Hence, the map $V_{N}: y \rightarrow v_{y}$ is an injective linear map from $N_{\sim}^{\perp}$ into itself. So

$$
\Phi(x \otimes y)=U_{N} x \otimes V_{N} y, \quad \forall x \in N, y \in N_{\sim}^{\perp} .
$$

Proposition 3.1. Suppose that $\mathcal{U}, \mathcal{V}$ are norm closed AlgN-modules, and that $\Phi$ : $\mathcal{U} \rightarrow \mathcal{V}$ is a module isomorphism. Then there exists a non-zero complex number $\lambda$ such that $\Phi(S)=\lambda S, \forall S \in \mathcal{O}(\mathcal{U})$. 
Proof. For each $N \in \mathcal{N}_{0}$, it follows from Lemma 3.4 that there exist injective linear maps $U_{N}: N \rightarrow N$ and $V_{N}: N_{\sim}^{\perp} \rightarrow N_{\sim}^{\perp}$ such that

$$
\Phi(x \otimes y)=U_{N} x \otimes V_{N} y, \quad \forall x \in N, y \in N_{\sim}^{\perp} .
$$

Suppose that $N_{1}, N_{2} \in \mathcal{N}_{0}$ and $N_{1}<N_{2}$, which follows from the definition of $N_{\sim}$ that $N_{1 \sim} \leq N_{2 \sim}$. Thus, for each $x \in N_{1}$ and $y \in N_{2 \sim}^{\perp}$, we have that

$$
\Phi(x \otimes y)=U_{N_{1}} x \otimes V_{N_{1}} y=U_{N_{2}} x \otimes V_{N_{2}} y .
$$

Hence there exist non-zero numbers $\xi, \eta$ such that $\xi \bar{\eta}=1$ and

$$
U_{N_{1}}=\left.\xi U_{N_{2}}\right|_{N_{1}},\left.\quad V_{N_{1}}\right|_{N_{2 \sim}^{\perp}}=\eta V_{N_{2}} .
$$

We now choose and fix a subspace $N_{0} \in \mathcal{N}_{0}$. For each $N \in \mathcal{N}_{0}$, the maps $U_{N}$ and $V_{N}$ may be normalized in such a way that they are equal to $U_{N_{0}}$ and $V_{N_{0}}$ on common domain respectively. Thus, we can define the linear maps

$$
U: \bigcup\left\{N: N \in \mathcal{N}_{0}\right\} \longrightarrow \bigcup\left\{N: N \in \mathcal{N}_{0}\right\},\left.\quad U\right|_{N}=U_{N}, \quad \forall N \in \mathcal{N}_{0},
$$

and

$$
V: \bigcup\left\{N_{\sim}^{\perp}: N \in \mathcal{N}_{0}\right\} \longrightarrow \bigcup\left\{N_{\sim}^{\perp}: N \in \mathcal{N}_{0}\right\},\left.\quad V\right|_{N_{\sim}^{\perp}}=V_{N}, \quad \forall N \in \mathcal{N}_{0}
$$

For each $x \otimes y \in \mathcal{U}$, it follows from [4, Lemma 1.1 and Lemma 1.3, that there exists $N \in \mathcal{N}_{0}$ such that $x \in N$ and $y \in N_{\sim}^{\perp}$. Hence

$$
\Phi(x \otimes y)=U_{N} x \otimes V_{N} y=U x \otimes V y .
$$

In the following, we will prove that the linear maps $U, V$ are trivial. For each $A \in \mathcal{A} \subseteq \operatorname{Alg} \mathcal{N}, N \in \mathcal{N}_{0}, x \in N$ and $y \in N_{\sim}^{\perp}$, we have that $\Phi(A x \otimes y)=A \Phi(x \otimes y)$ and $A x \in N$. Hence

$$
U_{N} A x \otimes V_{N} y=A U_{N} x \otimes V_{N} y, \quad \forall x \in N, y \in N_{\sim}^{\perp}
$$

and

$$
U_{N} A x=A U_{N} x, \quad \forall x \in N .
$$

So the linear map $U_{N}: N \rightarrow N$ commutes with $P(N) \mathcal{A P}(N)$, the algebra being interpreted as acting on the space $N$. Since $P(N) \mathcal{A} P(N)$ is a maximal abelian *-algebra in $\mathcal{B}(N)$, it follows from [9], Lemma 2.12, that $U_{N}$ is continuous. Now for each $T \in \operatorname{Alg} \mathcal{N}$, the same argument shows that

$$
U_{N} T x=T U_{N} x, \quad \forall x \in N .
$$

This shows that the bounded operator $U_{N}$ in $\mathcal{B}(N)$ commutes with the nest algebra $P(N) \operatorname{Alg} \mathcal{N} P(N)=\operatorname{Alg}(\mathcal{N} \cap N)$. Since the commutant of a nest algebra is trivial (see [1, Lemma 3.6), there exists a non-zero number $\lambda_{N} \in \mathbf{C}$ such that $U_{N}=$ $\lambda_{N} P(N)$. Similarly, there exists a non-zero number $\mu_{N} \in \mathbf{C}$ such that $V_{N}=$ $\mu_{N} P\left(N_{\sim}^{\perp}\right)$. For each $N \in \mathcal{N}_{0}$, since $U_{N}$ and $V_{N}$ are equal to $U_{N_{0}}$ and $V_{N_{0}}$ on common domain respectively, we obtain that $\lambda_{N}=\lambda_{N_{0}}$ and $\mu_{N}=\mu_{N_{0}}$. Therefore,

$$
U x=\lambda_{N_{0}} x, \quad \forall x \in \bigcup\left\{N: N \in \mathcal{N}_{0}\right\}
$$

and

$$
V y=\mu_{N_{0}} y, \quad \forall y \in \bigcup\left\{N_{\sim}^{\perp}: N \in \mathcal{N}_{0}\right\} .
$$

Let $\lambda=\lambda_{N_{0}} \bar{\mu}_{N_{0}}$. For each $x \otimes y \in \mathcal{U}$, it follows from the equation (6) that

$$
\Phi(x \otimes y)=\lambda_{N_{0}} \bar{\mu}_{N_{0}} x \otimes y=\lambda x \otimes y .
$$


Now we will give the main result of this paper.

Theorem 3.1. Suppose that $\mathcal{U}$ and $\mathcal{V}$ are norm closed AlgN-modules, and that $\Phi: \mathcal{U} \rightarrow \mathcal{V}$ is a module isomorphism. Then $\mathcal{U}=\mathcal{V}$ and there exists a number $\lambda$ such that $\Phi(T)=\lambda T, \forall T \in \mathcal{U}$.

Proof. It follows from Proposition 3.1 that there exists a non-zero number $\lambda$ such that

$$
\Phi(S)=\lambda S, \quad \forall S \in \mathcal{O}(\mathcal{U})
$$

For each $T \in \mathcal{U}$, we consider two cases separately.

Case 1. $\mathcal{H} \neq \mathcal{H}_{-}$. For each non-zero vector $x \in \mathcal{H}$ and $y \in \mathcal{H} \ominus \mathcal{H}_{-}$, the rank one operator $x \otimes y \in \operatorname{Alg} \mathcal{N}$. Hence,

$$
\Phi(T) x \otimes y=\Phi(T x \otimes y)=\lambda T x \otimes y .
$$

Therefore, we have

$$
\begin{gathered}
\Phi(T) x=\lambda T x, \quad \forall x \in \mathcal{H}, \\
\Phi(T)=\lambda T .
\end{gathered}
$$

Case 2. $\mathcal{H}=\mathcal{H}_{-}$. Choose an increasing sequence $\left\{N_{k}\right\} \subseteq \mathcal{N}, N_{k} \neq \mathcal{H}$ and $P\left(N_{k}\right) \stackrel{s}{\rightarrow} I$. For each $x \in \mathcal{H}, 0 \neq y_{k} \in N_{k}^{\perp}$, we have that $P\left(N_{k}\right) x \otimes y_{k} \in \operatorname{Alg} \mathcal{N}$. So

$$
\begin{gathered}
\Phi(T) P\left(N_{k}\right) x \otimes y_{k}=\Phi\left(T P\left(N_{k}\right) x \otimes y_{k}\right)=\lambda T P\left(N_{k}\right) x \otimes y_{k}, \\
\Phi(T) P\left(N_{k}\right) x=\lambda T P\left(N_{k}\right) x, \quad \forall x \in \mathcal{H} .
\end{gathered}
$$

By taking a limit, we obtain that

$$
\begin{gathered}
\Phi(T) x=\lambda T x, \quad \forall x \in \mathcal{H}, \\
\Phi(T)=\lambda T .
\end{gathered}
$$

From Case 1 and Case 2, it follows that $\Phi(T)=\lambda T, \forall T \in \mathcal{U}$. Thus we have $\mathcal{U}=\mathcal{V}$, and this completes the proof.

Theorem 3.1 shows that different norm closed $\operatorname{Alg} \mathcal{N}$-modules cannot be module isomorphic.

\section{REFERENCES}

1. E.Christensen, Derivations of nest algebras, Math. Ann. 229 (1977), 155-161. MR0448110 (56:6420)

2. J.A.Erdos, On certain elements of $C^{*}$-algebras, Illinois J. Math. 15 (1971), 682-693. MR0290120 $(44: 7305)$

3. J.A.Erdos, S. Giotopoulos and M.S.Lambrou, Rank one elements, Mathematika 24 (1977), 178-181. MR0467317(57:7176)

4. J.A.Erdos and S.C.Power, Weakly closed ideals of nest algebras, J. Operator Theory 7 (1982), 219-235. MR0658610 (84a:47056)

5. M.S.Lambrou, Automatic continuity and implementation of homomorphisms (manuscript).

6. M.S.Lambrou, On the rank one operators in reflexive algebras, Linear Alg. Applic. 142 (1990), 211-235. MR:1077986 (91k:47104)

7. W.E.Longstaff, Strongly reflexive lattices, J. London Math. Soc. 11 (1975), 491-512. MR0394233 (52:15036) 
8. W.E.Longstaff and Oreste Panaia, On the ranks of single elements of reflexive operator algebras, Proc. Amer. Math. Soc. 125 (1997), 2875-2882. MR.1402872 (97m:47061)

9. J.R.Ringrose, On some algebras of operators II, Proc. London Math. Soc. 16 (3) (1966), 385402. MR0196516(33:4703)

Department of Mathematics, Zhejiang University, Hangzhou, 310027, People's RePUBLIC OF CHINA

E-mail address: dongzhe@zju.edu.cn 